\title{
4. Erwiderung auf Hrn. Lenards Vorbemerkungen zur Soldnerschen Arbeit von $1801^{1}$ ); \\ von $M$. v. Laue.
}

1. Zum Hauptgegenstande können wir uns kurz fassen und ohne auf eine zurzeit noch aussichtslose Erörterung über den empirischen Betrag der Lichtablenkung an der Sonne einzugehen, die Frage stellen: Sollte nicht ein gewisser Fortschritt gegenüber Soldners Berechnung allein schon darin liegen, daB die allgemeine Relativitätstheorie diese Ablenkung mit der elektromagnetischen Wellentheorie ${ }^{2}$ ), statt mit der Emissionstheorie des Lichtes verknüpft?

Hr. Len ard behandelt daneben noch andere Themen. Wir greifen einige davon heraus und erwidern;

2. Die Trägheit der elektromagnetischen Energie war in der Tat schon vor der Relativitätstheorie bekannt. H. Poin$\left.\operatorname{car}^{3}\right)$ und M. Abraham ${ }^{4}$ ) haben sich wohl die gröBten Verdienste um diese Erkenntnis erworben, indem sie den Begriff des elektromagnetischen Impulses schufen. F. Hasenöhrl hat nur das - allerdings nicht gering zu schätzende - Verdienst des ersten Versuchs, aus diesem Begriff eine Dynamik der Hohlraumstrahlung herzuleiten. ${ }^{5}$ ) DaB aber jede Energieströmung Impuls mit sich führt, und $\mathrm{da} B$ umgekehrt aller Impuls anf Energieströmung beruht, diesen Gedanken konnte erst die Relativitätstheorie folgerichtig durchführen; denn erst sie räumte mit der ihm widersprechenden $\mathrm{New}$ tonschen Dynamik grundsätzlich auf. Die Masse, welche auf Energie beruht, nach einem

1) J. Soldner, Ann. d. Phys. 65. S. 593. 1921.

2) Vielleicht am deutlichsten bei M. v. Lane, Physik. Zeitschr. 21. S. 659.1920 .

3) H. Poincaré, Lorentz-Festschrift 1900. S. 252.

4) M. Abraham, Ann. d. Phys. 10. S. 105. 1903.

5) Richtig durchgeführt hat diese Betrachtungen erst die von Planck verbesserte Mosengeilsche Dissertation, Ann. d. Phys. 22. S. 867. 1907. 
bestimmten Autor zu benennen, wie Hr. Lenard vorschlägt, wäre gänzlich überflüssig, weil es nach unserer Auffassung welche auch $\mathrm{Hr}$. Lenard zu vertreten scheint, andersartige Masse überbaupt nicht gibt.

3. Hr. Lenard verweist auf seine Ableitung derjenigeri Dynamik, welche man gemeinhin als relativistisch bezeichnet. Nach dieser Berechnung ${ }^{1}$ ) soll sich die Kraft auf einen Massenpunkt mit der Geschwindigkeit $v$ zusammensetzen aus einem universellen Faktor $f(v)$ und einem Faktor $K_{0}$, welcher die Kraft unter sonst gleichen Umständen, aber für $v=0$, anzeigt. Wir ziehen daraus die Folgerung: „Die Arbeit, die ein elektrostatisches Feld leistet, wenn man eine Punktladung eine bestimmte Potentialdifferenz durchlaufen läBt, ist von der Durchlaufungsgeschwindigkeit abhängig“". Und weiter: „LäBt man die Punktladung in solchem Felde einen bestimmten Weg hin mit anderer Geschwindigkeit durchlaufen, als zurück, so hat man bei diesem KreisprozeB ein von Null verschiedenes Arbeitsquantum gewonnen."

4. In Anm. 2 zu S. 599 wirft Hr. Lenard die berechtigte Frage auf, weshalb man die Elektronenbewegung im Atom nach der beschränkten, und nicht nach der allgemeinen Relativitätstheorie quantelt. Die den theoretischen Physikern schon bekannte, aber vielleicht bisher nicht gedruckte Antwort lautet: Weil das keinen merklichen Unterschied in den GröBen der Terme ausmacht, und man mit den bis jetzt bekannten Beobachtungsmethoden, einschlieBlich der spektroskopischen, andere Eigenschaften der Quantenbahnen, als ihre Energie, d. h. die Tenne, nicht fassen kann. Die Begründung dafür findet sich in $\S 25$ von Band II meines Buches „Die Relativitätstheorie“ ${ }^{3}{ }^{3}$ )

Berlin, Oktober 1921.

1) P. Lenard, Jahrb. d. Radioaktivität und Elektronik 17. S. 307. 1921 ; vgl. S. 341 unten.

2) Erschienen Braunschweig 1921.

(Eingegangen 10. Oktober 1921.) 